\title{
Undecidability of the Transitive Graded Modal Logic with Converse
}

\author{
Evgeny Zolin \\ Faculty of Mathematics and Mechanics, \\ Moscow State University, Russia \\ e-mail: ezolin@gmail.com
}

\begin{abstract}
We extend the language of the modal logic $\mathbf{K 4}$ of transitive frames with two sorts of modalities. In addition to the usual possibility modality (which means that a formula holds in some successor of a given point), we introduce graded modalities (a formula holds in at least $n$ successors) and graded inverse modalities (a formula holds in at least $n$ predecessors). We show that the resulting logic, called GrIK4, is undecidable. The same result is obtained for all logics between GrIK4 and GrIS4. As a consequence, for the "unrestricted version" of the description logic $\mathcal{S I} \mathcal{Q}$, the problem of concept satisfiability (even with respect to the empty terminology) is undecidable. We also give a survey of complexity results for the local and global satisfiability problems for fragments of the logic GrIK4.
\end{abstract}

Keywords: modal logic, tense logic, graded modalities, description logic.

\section{Introduction}

Recent development in computational aspects of modal logic is to a certain extent motivated by its application in computer science, in particular, in artificial intelligence. This paper belongs to this trend of research, for its origin belongs to the field of knowledge representation, or more specifically, of description logics (DLs) [1]. In order to formulate our result, we need to specify three things: the modal language, the class of Kripke frames, and the decision problem. All three components have their natural counterparts in DLs (for details, see Section 5).

Recall that the standard modal language has the "possibility" modality $\diamond$; a formula $\diamond A$ at a point $x$ of a model means that "there is a successor of $x$ in which the formula $A$ is true". We augment this language with the so-called graded modalities $\diamond^{\geqslant n}$, which mean "there are at least $n$ successors of $x \ldots$., inverse (also called converse) modalities $\diamond$ ("there is a predecessor of $x \ldots$.), and graded inverse modalities $\theta^{\geqslant n}$ ("there are at least $n$ predecessors of $x \ldots$ ").

The class of frames we consider is the class of all transitive frames. In addition, the class of all reflexive transitive frames is considered as well.

Two decision problems are addressed in this paper: the local satisfiability of formulas of some modal language in some class of frames $\mathcal{F}$ : given a formula $A$, determine whether it is true at some point of some model based on some frame from $\mathcal{F}$, and the global satisfiability: given a formula $A$, determine whether it is true in some model based on some frame from $\mathcal{F}$. Our main result is that, for the above modal language and the class of (reflexive) transitive frames, both problems are undecidable.

Closely related is the "unrestricted" DL $\mathcal{S I} \mathcal{Q}$ [7] (see also Section 5), where by "unrestricted" we mean that transitive roles are not forbidden in number restrictions. Our results imply that, 
for this DL, the problems of concept satisfiability and of terminology consistency are undecidable, even if there is only one transitive role in the vocabulary. Note that, for this DL, the (more general and hence more difficult) problem of concept satisfiability w.r.t. terminologies was shown to be undecidable in [9]. To the best of our knowledge, these are the first undecidability results obtained for any fragment of $\mathcal{S H O I Q}$ - the DL that underpins the Web Ontology language, OWL, see [10] - in absence of role hierarchy.

The method of proof deserves a few words. We prove the undecidability by reduction from the undecidable domino problem for $\mathbb{Z} \times \mathbb{Z}$. Typically, to obtain such a reduction for a given logic $\mathbf{L}$, one constructs a model $M$ that 'resembles' $\mathbb{Z} \times \mathbb{Z}$, then builds a formula $A$, and proves that this formula helps to reduce the domino problem to a given decision problem for $\mathbf{L}$. However, in such a proof, the exact relationship between the logic $\mathbf{L}$, the model $M$, and the formula $A$ remains implicit.

We make it explicit by introducing the following notion: a model $M$ is expressed by a formula $A$ in a logic $\mathbf{L}$. Intuitively, this means that $M$ is an $\mathbf{L}$-model, satisfies $A$, and is embeddable into every L-model satisfying $A$. This makes it possible to split the undecidability proof into two loosely related parts. In the first part, one shows that a particular model (that looks like $\mathbb{Z} \times \mathbb{Z}$ ) is expressible in the given logic by some formula. In the second part, one proves that if this model is expressible in any logic by any modal formula, then this suffices for the logic to be undecidable.

The paper is organized as follows. After giving necessary definitions and fixing notation in Section 1, we introduce, in Section 2, the local and the global versions of the notion "a model is expressible in a modal logic." Using this notion, we first prove the global undecidability of the modal logic GrIK4 in Section 3, and then prove its local undecidability in Section 4. In parallel, we prove the same results for the logic GrIS4, the reflexive counterpart of GrIK4. In Section 5, we discuss a close relationship between the modal logic GrIK4 and the "unrestricted" description logic $\mathcal{S I} \mathcal{Q}$ and show that, for the latter, the problems of concept satisfiability and the terminology consistency are undecidable. Section 6 presents a survey of decidability and complexity results for fragments of the logic GrIK4. Finally, we conclude the paper by discussing further directions of research.

\section{Preliminaries}

Syntax. The language of the graded modal logic with converse has countably many propositional variables $\left\{p_{0}, p_{1}, \ldots\right\}$, Boolean connectives $\perp, \rightarrow$ (others are taken as standard abbreviations) and two sorts of modal operators $\diamond^{\geqslant n}$ and $\vartheta^{\geqslant n}$, for all integers $n \geqslant 1$. Formulas are built up according to the syntax:

$$
A, B \quad::=\perp\left|p_{i}\right| A \rightarrow B\left|\diamond^{\geqslant n} A\right| \ominus^{\geqslant n} A .
$$

Some shortcuts are useful: $\diamond A:=\diamond^{\geqslant 1} A, \square A:=\neg \diamond \neg A, \diamond^{\leqslant n} A:=\neg \diamond^{\geqslant n+1} A$; similarly for $\diamond^{>n}$, $\diamond^{<n}, \diamond^{=n}$, and for $\diamond$, e.g. $\boxminus A=\neg \diamond \neg A$. Denote by $\operatorname{Var}(A)$ the set of variables occurring in $A$.

Semantics. A frame $F=(W, R)$ consists of a nonempty set of points $W$ and a binary accessibility relation $R \subseteq W \times W$. A model based on a frame $F$ is a pair $M=(F, \theta)$, where $\theta$ is a valuation that assigns to every variable $p$ a subset of points $\theta(p) \subseteq W$. The truth relation $M, x=A$ (where we usually omit $M$ ) is defined by induction on the construction of $A$ : $x \mid \neq \perp$; $x \models p$ iff $x \in \theta(p) ; x \models A \rightarrow B$ iff $x \not \models A$ or $x \models B$; finally, for the graded modal operators, we 
define: ${ }^{1}$

$$
\begin{aligned}
& x=\diamond^{\geqslant n} A \quad \text { iff } \quad \exists^{\geqslant n} y \in W: x R y \text { and } y=A, \\
& x=\diamond^{\geqslant n} A \quad \text { iff } \quad \exists^{\geqslant n} y \in W: y R x \text { and } y=A .
\end{aligned}
$$

Thus, $\diamond^{\geqslant n} A$ is true at a point $x$ if $A$ is true in at least $n$ successors of $x$, whereas $\diamond^{\geqslant n} A$ is true at $x$ if $A$ is true in at least $n$ predecessors of $x$. We say that a formula $A$ is true in a model $M$ and write $M=A$ if $A$ is true at all points of $M$. A formula $A$ is valid on a frame $F$, written as $F \models A$, if it is true in all models based on $F$. These notions extend naturally to classes of models and frames and to sets of formulas.

\subsection{Modal logics}

A (modal) logic is a pair $\mathbf{L}=(\mathcal{L}, \mathcal{F})$, where $\mathcal{L}$ is a modal language (a set of modal operators, at least containing $\diamond$, with their semantics) and $\mathcal{F}$ is a class of frames. By an $\mathbf{L}$-formula we mean a formula in the language $\mathcal{L}$; an $\mathbf{L}$-model is a model $M=(F, \theta)$ with $F \in \mathcal{F}$.

Naming logics. The names $\mathbf{K}, \mathbf{K} 4, \mathbf{S} 4$ stand for the logics in the language $\mathcal{L}=\{\diamond\}$ of the classes of all frames, all transitive frames, and all reflexive transitive frames, respectively. We denote logics in extended languages by adding a prefix to their names:

- the prefix $\mathbf{G r}$ stands for the language $\mathcal{L}=\left\{\diamond^{\geqslant n} \mid n \geqslant 1\right\}$;

- the prefix I stands for the language $\mathcal{L}=\{\diamond, \diamond\}$;

- the prefix GrI stands for the language $\mathcal{L}=\left\{\diamond^{\geqslant n}, \diamond^{\geqslant n} \mid n \geqslant 1\right\}$.

For example, the logic GrIK4 is determined by the set of operators $\mathcal{L}=\left\{\diamond^{\geqslant n}, \diamond^{\geqslant n} \mid n \geqslant 1\right\}$ (with the semantics defined above) and the class of all transitive frames.

Fragments and sublogics. Let $\mathbf{L}=(\mathcal{L}, \mathcal{F})$ and $\mathbf{L}^{\prime}=\left(\mathcal{L}^{\prime}, \mathcal{F}^{\prime}\right)$ be two logics.

- $\mathbf{L}$ is called a fragment of $\mathbf{L}^{\prime}$ if $\mathcal{L} \subseteq \mathcal{L}^{\prime}$ and $\mathcal{F}=\mathcal{F}^{\prime}$.

- $\mathbf{L}$ is called a sublogic of $\mathbf{L}^{\prime}$ if $\mathcal{L}=\mathcal{L}^{\prime}$ and $\mathcal{F} \supseteq \mathcal{F}^{\prime}$.

We write $\mathbf{L} \subseteq \mathbf{L}^{\prime}$ if both $\mathcal{L} \subseteq \mathcal{L}^{\prime}$ and $\mathcal{F} \supseteq \mathcal{F}^{\prime}$. This notion generalizes the usual inclusion between logics to the case of extending the language of a logic. Note that if we identify a $\operatorname{logic} \mathbf{L}=(\mathcal{L}, \mathcal{F})$ with the set of its validities ${ }^{2}$ (i.e., the set of $\mathcal{L}$-formulas that are valid on $\mathcal{F}$ ), then the relation $\mathbf{L} \subseteq \mathbf{L}^{\prime}$ coincides with the usual set-theoretic inclusion. For example, we have the following inclusions:

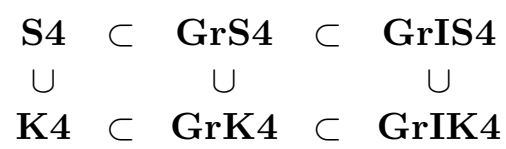

Satisfiability. Given a $\operatorname{logic} \mathbf{L}=(\mathcal{L}, \mathcal{F})$, an $\mathbf{L}$-formula $A$ is said to be

- locally satisfiable in $\mathbf{L}$ if $A$ is true at some point of some $\mathbf{L}$-model;

- globally satisfiable in $\mathbf{L}$ if $A$ is true in some $\mathbf{L}$-model.

\footnotetext{
${ }^{1}$ Here we use an abbreviation: $\exists^{\geqslant n} y \Phi(y):=\exists y_{1}, \ldots, y_{n}\left(\bigwedge_{i \neq j} y_{i} \neq y_{j} \wedge \bigwedge_{i=1}^{n} \Phi\left(y_{i}\right)\right)$.

${ }^{2}$ This means that we consider only Kripke complete modal logics.
} 
We call a logic L locally (resp., globally) decidable if the problem of local (resp., global) satisfiability of formulas in $\mathbf{L}$ is decidable.

Usually one also introduces the notion "a formula $A$ is valid in $\mathbf{L}$ ", which means that $\mathcal{F} \models A$. It is easily seen that validity is dual to local satisfiability: $A$ is valid in $\mathbf{L}$ iff $\neg A$ is not locally satisfiable in $\mathbf{L}$. Therefore, if we identify a logic with the set of its validities, then the notion of local decidability of a logic coincides with the usual notion of decidability (for this reason, we often omit the word 'locally'). In general, the problems of global and local satisfiability are not reducible to each other, for the global one is for some logics harder and for some other logics easier than the local one, as Table 1 on p. 14 illustrates. The notion of local satisfiability (and its dual, validity) has been extensively explored in modal logic. On the contrary, the global satisfiability have received much less attention. However, it is the latter notion (and a more general one, the global consequence relation, not considered here) that plays a crucial rôle for knowledge representation and reasoning in description logics (many of which are notational variants of modal logics); see Section 5 .

\subsection{Domino problem}

Our undecidability proofs are given by reduction from the undecidable "domino problem".

Definition 1.1. A domino system $\mathcal{D}=(D, H, V)$ consists of a finite set $D=\left\{d_{1}, \ldots, d_{\kappa}\right\}$ of tile types and horizontal and vertical matching relations $H, V \subseteq D \times D$. A domino system $\mathcal{D}$ tiles $\mathbb{Z} \times \mathbb{Z}$ if there exists a $\mathcal{D}$-tiling, i.e., a total function $t: \mathbb{Z} \times \mathbb{Z} \rightarrow D$ satisfying the following compatibility constraints: $\langle t(m, n), t(m+1, n)\rangle \in H$ and $\langle t(m, n), t(m, n+1)\rangle \in V$, for all $m, n \in \mathbb{Z}$. The domino problem for $\mathbb{Z} \times \mathbb{Z}$ is to determine whether a given domino system $\mathcal{D}$ tiles $\mathbb{Z} \times \mathbb{Z}$.

In other words, given a domino system $\mathcal{D}$, the problem is to check whether copies of tiles of the given types $d_{1}, \ldots, d_{\kappa}$ can be placed on the $\mathbb{Z} \times \mathbb{Z}$ grid so that horizontally and vertically adjacent tiles comply with the given relations $H$ and $V$.

Theorem 1.1 (Berger, 1966, see e.g. [4]). The domino problem for $\mathbb{Z} \times \mathbb{Z}$ is undecidable.

If the domino problem is reduced to the (local or global) satisfiability for two logics $\mathbf{L}_{1}$ and $\mathbf{L}_{2}$, where $\mathbf{L}_{1} \subset \mathbf{L}_{2}$, and the reduction uses the same modal formulas, then we immediately obtain the undecidability result for all logics in the interval $\left[\mathbf{L}_{1}, \mathbf{L}_{2}\right]$, as the following lemma shows.

Lemma 1.2. Let $\mathbf{L}_{1} \subset \mathbf{L}_{2}$ be two logics. Assume that one can effectively build, given a domino system $\mathcal{D}$, an $\mathbf{L}_{1}$-formula $\Phi_{\mathcal{D}}$ such that the following statements are equivalent:

(i) $\mathcal{D}$ tiles $\mathbb{Z} \times \mathbb{Z}$;

(ii) the formula $\Phi_{\mathcal{D}}$ is satisfiable in $\mathbf{L}_{1}$;

(iii) the formula $\Phi_{\mathcal{D}}$ is satisfiable in $\mathbf{L}_{2}$.

Then every logic $\mathbf{L}$ with $\mathbf{L}_{1} \subseteq \mathbf{L} \subseteq \mathbf{L}_{2}$ is undecidable. Similarly for the global satisfiability.

Proof. Simply add the following statement to the above three:

(ii') the formula $\Phi_{\mathcal{D}}$ is satisfiable in $\mathbf{L}$.

Obviously, the implications $(i i i) \Rightarrow\left(i i^{\prime}\right) \Rightarrow(i i)$ hold, so all the four statements are equivalent. Thus, the mapping $\mathcal{D} \mapsto \Phi_{\mathcal{D}}$ yields the required reduction. 


\section{Expressing a model in a logic}

Our reduction of the domino problem to the (local or global) satisfiability problem in a given logic follows the standard pattern: given a domino system, we effectively build a formula such that it is (locally or globally) satisfiable in the logic if, and only if, the domino system tiles the grid $\mathbb{Z} \times \mathbb{Z}$. The way we present the proof reveals, in a sense, the "reason" why the given logic is undecidable. For this, we introduce the (local and global versions of the) notion "a model is expressed by a formula in a logic." This allows us to divide the undecidability proof into two loosely related parts that might be interesting on their own right.

Firstly, some model (that looks like a grid) is shown to be (locally or globally) expressible by some modal formula in a given logic. Secondly, it is shown that every logic in which this model is (locally or globally) expressible is (locally or globally) undecidable; this is achieved by a reduction from the domino problem.

Let us first introduce the "global" version of the notion and then its local counterpart.

\subsection{Global expressibility of models}

Let $\mathbb{P} \subset\left\{p_{0}, p_{1}, \ldots\right\}$ be a finite set of variables. A model over $\mathbb{P}$ is a pair $M=(F, \theta)$, where $F$ is a frame and $\theta$ a valuation of variables from $\mathbb{P}$ only, i.e., a function $\theta: \mathbb{P} \rightarrow 2^{W}$.

Let $M=(W, R, \theta)$ be a model over $\mathbb{P}$ and $M^{\prime}=\left(W^{\prime}, R^{\prime}, \theta^{\prime}\right)$ be an ordinary model.

Definition 2.1. A homomorphism from $M$ to $M^{\prime}$ is a mapping $h$ : $W \rightarrow W^{\prime}$ that satisfies the following two conditions, for all points $x, y \in W$ and all variables $p \in \mathbb{P}$ :

(1) $x R y \Longrightarrow h(x) R^{\prime} h(y)$;

(2) $M, x \models p \Longleftrightarrow M^{\prime}, h(x) \models p$.

We say that $M$ is embeddable into $M^{\prime}$, written as $M \hookrightarrow M^{\prime}$, if there is a homomorphism ${ }^{3}$ from $M$ to $M^{\prime}$.

Now let $\mathbf{L}=(\mathcal{L}, \mathcal{F})$ be a logic, $A$ an $\mathbf{L}$-formula with $\operatorname{Var}(A)=\mathbb{P}$, and $M$ a model over $\mathbb{P}$.

Definition 2.2. The model $M$ is (globally) expressed in the logic $\mathbf{L}$ by the formula $A$ if the following conditions hold (intuitively, $M$ is the "minimal" (w.r.t. $\hookrightarrow$ ) $\mathbf{L}$-model of $A$ ):

(i) $M$ is an $\mathbf{L}$-model, i.e., it is based on a frame from $\mathcal{F}$,

(ii) $M \models A$,

(iii) for every model $M^{\prime}$ satisfying (i) and (ii), we have $M \hookrightarrow M^{\prime}$.

We say that a model $M$ over $\mathbb{P}$ is (globally) expressible in $\mathbf{L}$ if it is expressed in $\mathbf{L}$ by some L-formula $A$ with $\operatorname{Var}(A)=\mathbb{P}$.

In order to prove the global undecidability of some logic $\mathbf{L}_{0}$, we will build a model $M_{0}$ (over some finite set of variables $\mathbb{P}$ ) that satisfies the following two properties:

Embedding. The model $M_{0}$ is globally expressible in the logic $\mathbf{L}_{0}$.

Reduction. If the model $M_{0}$ is globally expressible in any logic $\mathbf{L}$ (that has the modal operator $\diamond$ in its language), then $\mathbf{L}$ is globally undecidable.

\footnotetext{
${ }^{3}$ Note that we do not require a homomorphism to be injective or surjective.
} 


\subsection{Local expressibility of pointed models}

A pointed model is a pair $(M, w)$, where $M$ is a model and $w$ is a point in it. A pointed model over $\mathbb{P}$ and a pointed $\mathbf{L}$-model are defined in the obvious way. Let $(M, w)$ be a pointed model over $\mathbb{P}$ and and $\left(M^{\prime}, w^{\prime}\right)$ an ordinary pointed model.

Definition 2.3. A homomorphism from $(M, w)$ to $\left(M^{\prime}, w^{\prime}\right)$ is a homomorphism from $M$ to $M^{\prime}$ (as per Definition 2.1) that sends $w$ to $w^{\prime}$. We say that $(M, w)$ is embeddable into $\left(M^{\prime}, w^{\prime}\right)$, written as $(M, w) \hookrightarrow\left(M^{\prime}, w^{\prime}\right)$, if there is a homomorphism from $(M, w)$ to $\left(M^{\prime}, w^{\prime}\right)$.

Now let $\mathbf{L}=(\mathcal{L}, \mathcal{F})$ be a logic, $A$ an $\mathbf{L}$-formula with $\operatorname{Var}(A)=\mathbb{P}$, and $(M, w)$ a pointed model over $\mathbb{P}$.

Definition 2.4. The pointed model $(M, w)$ is (locally) expressed in the logic $\mathbf{L}$ by the formula $A$ if the following conditions hold:

(i) $(M, w)$ is a pointed $\mathbf{L}$-model, i.e., $M$ is based on a frame from $\mathcal{F}$,

(ii) $M, w \models A$,

(iii) for every pointed model $\left(M^{\prime}, w^{\prime}\right)$ satisfying $(i)$ and $(i i)$, we have $(M, w) \hookrightarrow\left(M^{\prime}, w^{\prime}\right)$.

We say that the pointed model $(M, w)$ over $\mathbb{P}$ is (locally) expressible in $\mathbf{L}$ if it is expressed in $\mathbf{L}$ by some $\mathbf{L}$-formula $A$ with $\operatorname{Var}(A)=\mathbb{P}$.

The proof of the local undecidability of a logic $\mathbf{L}_{0}$ will follow the same pattern as for the global case (i.e., embedding and reduction), but with pointed models instead of models, and 'locally' instead of 'globally' everywhere.

\section{Global undecidability of GrIK4}

Our first result is that the problem of global satisfiability of formulas in the logic GrIK4 is undecidable. Following the pattern described above, the proof consists of two parts. First, a model "similar" to $\mathbb{Z} \times \mathbb{Z}$ is shown to be expressible in GrIK4. Global satisfiability of formulas is well suited for this purpose, as it allows one to impose necessary constraints on all points of a model. Secondly, we use this expressibility fact in order to reduce the domino problem to the global satisfiability problem. At the end of the section, we show that the construction works for all logics between GrIK4 and GrIS4.

\subsection{Expressing a grid}

Let $\mathbb{P}=\left\{p_{i j} \mid 0 \leqslant i, j \leqslant 3\right\}$ and consider the model $\mathfrak{M}$ depicted in Fig. 1. Informally, we place the 16 variables $p_{i j}$ onto the $\mathbb{Z} \times \mathbb{Z}$ grid (by periodically repeating the $[0,3] \times[0,3]$ pattern) and link the points as shown in Fig. 1 . Formally, we have $\mathfrak{M}=(\mathbb{Z} \times \mathbb{Z}, \prec, \lambda)$, where $\prec$ is the transitive closure of the following relation Edges depicted in Fig. 1:

$$
\begin{aligned}
\text { Edges } & =\text { Right } \cup \text { Left } \cup \text { Up } \cup \text { Down, } \\
\text { Right } & =\{\langle(m, n),(m+1, n)\rangle \mid m, n \in \mathbb{Z}, m \text { is even }\}, \\
\text { Left } & =\{\langle(m, n),(m-1, n)\rangle \mid m, n \in \mathbb{Z}, m \text { is even }\}, \\
\text { Up } & =\{\langle(m, n),(m, n+1)\rangle \mid m, n \in \mathbb{Z}, n \text { is even }\}, \\
\text { Down } & =\{\langle(m, n),(m, n-1)\rangle \mid m, n \in \mathbb{Z}, n \text { is even }\}, \\
\lambda\left(p_{i j}\right) & =\{\langle i+4 m, j+4 n\rangle \mid m, n \in \mathbb{Z}\} .
\end{aligned}
$$

Denote by $\mathfrak{M}_{\bullet}=(\mathbb{Z} \times \mathbb{Z}, \preccurlyeq, \lambda)$ the reflexive closure of the model $\mathfrak{M}$. Now let $A$ be the conjunction of the following formulas, where $i, j, k, \ell$ range over $\{0,1,2,3\}$ : 


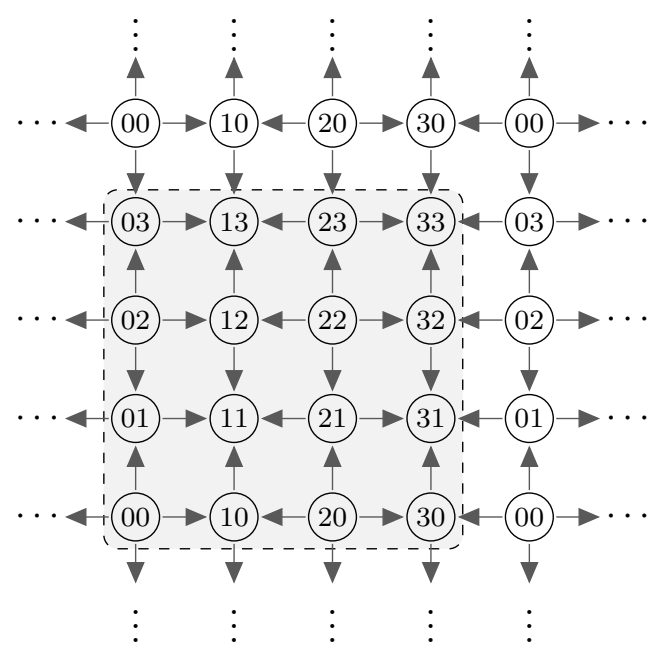

Figure 1: The $\mathbb{Z} \times \mathbb{Z}$-like model $\mathfrak{M}$ over the set of variables $\mathbb{P}=\left\{p_{i j} \mid 0 \leqslant i, j \leqslant 3\right\}$. Digits $(i j)$ in circles indicate which variable $p_{i j}$ is true at each point. The framed $4 \times 4$ pattern of points is repeated periodically over $\mathbb{Z} \times \mathbb{Z}$.

\begin{tabular}{|c|c|c|}
\hline (1) & $\bigvee_{i, j} p_{i j}$ & \\
\hline$(2)$ & $\neg\left(p_{i j} \wedge p_{k \ell}\right)$ & for all $\langle i, j\rangle \neq\langle k, \ell\rangle$ \\
\hline (3) & $\left.\begin{array}{l}p_{i j} \rightarrow \diamond p_{k \ell} \\
p_{k \ell} \rightarrow \diamond p_{i j}\end{array}\right\}$ & for all $\langle i, j\rangle \mapsto\langle k, \ell\rangle$ \\
\hline (4) & $\left.\begin{array}{l}p_{i j} \rightarrow \diamond^{\leqslant 1} p_{k \ell} \\
p_{k \ell} \rightarrow \diamond^{\leqslant 1} p_{i j}\end{array}\right\}$ & $\begin{array}{l}\text { for all } i, j \in\{0,2\} \\
\text { and all } k, \ell \in\{1,3\}\end{array}$ \\
\hline
\end{tabular}

Here $\langle i, j\rangle \Leftrightarrow\langle k, \ell\rangle$, for $0 \leqslant i, j, k, \ell \leqslant 3$, intuitively means that, in Fig. 1 , an arrow connects the point $(i j)$ to the point $(k \ell)$; formally, this relation is defined by the equivalence:

$$
\left\langle(m, n),\left(m^{\prime}, n^{\prime}\right)\right\rangle \in \text { Edges } \Longleftrightarrow\langle m \bmod 4, n \bmod 4\rangle \Leftrightarrow\left\langle m^{\prime} \bmod 4, n^{\prime} \bmod 4\right\rangle .
$$

Lemma 3.1. (a) The model $\mathfrak{M}$ is globally expressible in the logic GrIK4 (by the formula A).

(b) The model $\mathfrak{M}$. is globally expressible in the logic GrIS4 (by the same formula A).

Proof. We only prove (a); the proof of (b) is similar. Since $\prec$ is transitive, $\mathfrak{M}$ is a GrIK4model. It is not hard to see that $\mathfrak{M} \models A$. In particular, the formulas (4) are true in $\mathfrak{M}$ since, for example, from any point satisfying $p_{00}$ one can reach, via the relation $\prec$, only one point satisfying $p_{11}$, and only one point satisfying $p_{13}$, and so on.

It remains to show that the model $\mathfrak{M}$ is embeddable into every transitive model $M=$ $(W, R, \theta)$ that globally satisfies the formula $A$. To this end, we will find (not necessarily distinct) points $\left\{w_{m n} \in W \mid m, n \in \mathbb{Z}\right\}$ such that the function $h: \mathbb{Z} \times \mathbb{Z} \rightarrow W$ defined by $h(m, n)=w_{m n}$ is a homomorphism from $\mathfrak{M}$ to $M$.

First, we claim that $M$ has a point satisfying $p_{00}$. Indeed, $M$ contains at least one point $w$; by (1) it satisfies at least one of $p_{i j}$. If, for example, $w \models p_{12}$ (for other cases, the argument is similar), then, by (3), $w$ has an $R$-successor $w^{\prime}$ satisfying $p_{11} ; w^{\prime}$ in turn has an $R$-predecessor $w^{\prime \prime}$ satisfying $p_{10}$; finally, $w^{\prime \prime}$ has an $R$-predecessor $w^{\prime \prime \prime}$ satisfying $p_{00}$.

So, let $w_{00}$ be a point in $M$ such that $w_{00} \models p_{00}$. This point will be the "origin" of the grid. Next, using the formulas (3), we show that $M$ contains points that form a "horizontal axis" and 

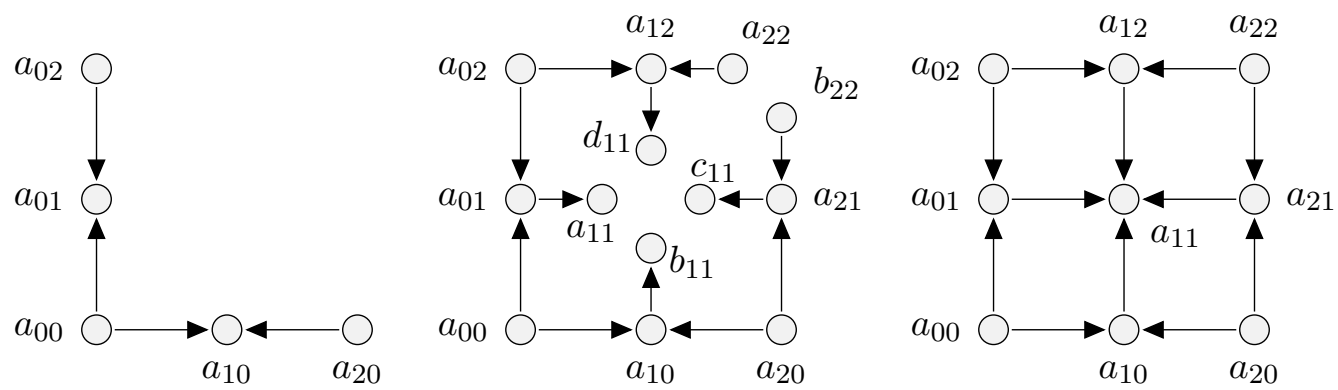

Figure 2: Building a $3 \times 3$ block of points.

a "vertical axis". Indeed,

- since $w_{00}=p_{00} \rightarrow \diamond p_{10}$, there is $w_{10} \in W$ such that $w_{00} R w_{10}$ and $w_{10}=p_{10}$,

- since $w_{10}=p_{10} \rightarrow \diamond p_{20}$, there is $w_{20} \in W$ such that $w_{20} R w_{10}$ and $w_{20}=p_{20}$,

- since $w_{20}=p_{20} \rightarrow \diamond p_{30}$, there is $w_{30} \in W$ such that $w_{20} R w_{30}$ and $w_{30}=p_{30}$,

and so on; similarly in the opposite direction. By induction, there exist points $\left\{w_{m, 0} \in W \mid\right.$ $m \in \mathbb{Z}\}$ that are linked by $R$-edges of interleaving directions:

$$
\ldots \stackrel{R}{\longleftarrow} w_{-2,0} \stackrel{R}{\longrightarrow} w_{-1,0} \stackrel{R}{\longleftarrow} w_{0,0} \stackrel{R}{\longrightarrow} w_{1,0} \stackrel{R}{\longleftarrow} w_{2,0} \stackrel{R}{\longrightarrow} \ldots
$$

and satisfy $w_{m, 0}=p_{i, 0}$, where $i=m \bmod 4$. Likewise, there exist points $\left\{w_{0, n} \in W \mid n \in \mathbb{Z}\right\}$ that are linked similarly and satisfy $w_{0, y}=p_{0, j}$, where $j=n \bmod 4$.

Now we "complete" the grid as follows. Whenever we have two horizontal and two vertical edges consisting of points $a_{00}, a_{10}, a_{20}, a_{01}, a_{02}$, as in Fig. 2(left), we complete them in a $3 \times 3$ block shown in Fig. 2(right). To this end, first we build a "pre-grid"; namely, by (3), there are 8 points (four points $a_{11}, b_{11}, c_{11}, d_{11}$, two points $a_{12}, a_{21}$, and two points $a_{22}, b_{22}$ ) that are linked as in Fig. 2(middle) and satisfy the variables $p_{i j}$ that correspond to their subscripts. Then we use the formulas (4) to "merge" points with the identical subscripts:

- since $a_{00}=p_{00} \rightarrow \diamond^{\leqslant 1} p_{11}$, we conclude that $a_{11}=b_{11}$,

- since $a_{20}=p_{20} \rightarrow \diamond^{\leqslant 1} p_{11}$, we conclude that $b_{11}=c_{11}$,

- since $a_{02} \models p_{02} \rightarrow \diamond^{\leqslant 1} p_{11}$, we conclude that $c_{11}=d_{11}$,

- since $a_{11} \models p_{11} \rightarrow \diamond^{\leqslant 1} p_{22}$, we conclude that $a_{22}=b_{22}$.

Thus we obtain a $3 \times 3$ grid of elements shown in Fig. 2(right). Continuing this process (in four directions), we can build the whole "grid" of points $\left\{w_{m n} \in W \mid m, n \in \mathbb{Z}\right\}$ that are linked as the corresponding nodes of the model $\mathfrak{M}$ shown in Fig. 1 , and that satisfy $w_{m n} \models p_{i j}$, where $i=m \bmod 4$ and $j=n \bmod 4$. It immediately follows from the above construction that the mapping $h: \mathbb{Z} \times \mathbb{Z} \rightarrow W$ defined by $h(m, n)=w_{m n}$ is a homomorphism from $\mathfrak{M}$ to $M$.

\subsection{Reducing the domino problem}

Recall that $\mathfrak{M}=(\mathbb{Z} \times \mathbb{Z}, \prec, \lambda)$ is the $\mathbb{Z} \times \mathbb{Z}$-like model depicted in Fig. 1 and $\mathfrak{M} \bullet=(\mathbb{Z} \times \mathbb{Z}, \preccurlyeq, \lambda)$ is its reflexive counterpart.

Lemma 3.2. Assume that the language of a logic $\mathbf{L}$ contains the modal operator $\diamond$.

(a) If the model $\mathfrak{M}$ is globally expressible in $\mathbf{L}$, then $\mathbf{L}$ is globally undecidable.

(b) If the model $\mathfrak{M}_{\bullet}$ is globally expressible in $\mathbf{L}$, then $\mathbf{L}$ is globally undecidable.

Proof. We only prove (a); the proof of (b) is similar (even with the same formulas). By assumption, the model $\mathfrak{M}$ is globally expressed in $\mathbf{L}$ by some $\mathcal{L}$-formula $A$. We will show that the 
domino problem is reducible to the problem of global satisfiability in $\mathbf{L}$. Given a domino system $\mathcal{D}=(D, H, V)$, to simplify notation, let us regard the elements of $D$ as propositional variables (not in $\mathbb{P}$ ). Let $A_{\mathcal{D}}$ be the conjunction of the following formulas, for all $i, j \in\{0,1,2,3\}$ and $c, d \in D$, where $\oplus$ and $\ominus$ are the addition and the subtraction modulo 4 :

\begin{tabular}{|cll|}
\hline (Partitioning) & $\begin{cases}\bigvee_{d \in D} d & \text { for } c \neq d \\
\neg(c \wedge d) & \text { for } i \in\{0,2\}\end{cases}$ \\
(Compatibility) & $\begin{cases}\left(p_{i j} \wedge c\right) \rightarrow \square\left(p_{i \oplus 1, j} \rightarrow \bigvee_{\langle c, d\rangle \in H} d\right) & \text { for } i \in\{0,2\} \\
\left(p_{i j} \wedge d\right) \rightarrow \square\left(p_{i \ominus 1, j} \rightarrow \bigvee_{\langle c, d\rangle \in H} c\right) & \text { for } j \in\{0,2\} \\
\left(p_{i j} \wedge c\right) \rightarrow \square\left(p_{i, j \oplus 1} \rightarrow \bigvee_{\langle c, d\rangle \in V} d\right) & \text { for } j \in\{0,2\} \\
\left(p_{i j} \wedge d\right) \rightarrow \square\left(p_{i, j \ominus 1} \rightarrow \bigvee_{\langle c, d\rangle \in V} c\right) & \end{cases}$
\end{tabular}

Claim. A domino system $\mathcal{D}$ tiles $\mathbb{Z} \times \mathbb{Z} \Longleftrightarrow$ the formula $A \wedge A_{\mathcal{D}}$ is globally satisfiable in $\mathbf{L}$. $(\Rightarrow)$ Given a $\mathcal{D}$-tiling $t: \mathbb{Z} \times \mathbb{Z} \rightarrow D$, let $M$ be the model that extends the model $\mathfrak{M}=(\mathbb{Z} \times \mathbb{Z}, \prec, \lambda)$ to the variables $d \in D$ by putting

$$
\lambda(d)=\{\langle m, n\rangle \in \mathbb{Z} \times \mathbb{Z} \mid t(m, n)=d\} .
$$

We claim that $M \models A \wedge A_{\mathcal{D}}$. Clearly, $M \models A$, since the formula $A$ expresses the model $\mathfrak{M}$ in $\mathbf{L}$ and hence $\mathfrak{M} \models A$. Furthermore, $M \models$ (Partitioning), since $t$ is a total function. Finally, let us prove that $M$ satisfies the first formula from (Compatibility); for the others, the argument is similar.

Take any $w=\langle m, n\rangle \in \mathbb{Z} \times \mathbb{Z}$. We show that, for each $i \in\{0,2\}, j \in\{0,1,2,3\}$, and $c \in D$,

$$
M, w \models\left(p_{i j} \wedge c\right) \rightarrow \square\left(p_{i \oplus 1, j} \rightarrow \bigvee_{\langle c, d\rangle \in H} d\right) .
$$

Assume that $\langle m, n\rangle \models p_{i j} \wedge c$. Then $i=m \bmod 4, j=n \bmod 4, t(m, n)=c$, and $m$ is even. Now take any $w^{\prime} \succ w$ with $w^{\prime} \models p_{i \oplus 1, j}$. By construction of $\mathfrak{M}$, this is only possible if $\left(w, w^{\prime}\right) \in$ Right, so that $w^{\prime}=\langle m+1, n\rangle$. Now put $d:=t(m+1, n)$. Then $\langle c, d\rangle \in H$, due to the horizontal compatibility condition for $t$, and $w^{\prime} \models d$.

$(\Leftarrow)$ Assume that $M=(W, R, \theta)$ is an arbitrary $\mathbf{L}$-model such that $M \models A \wedge A_{\mathcal{D}}$. Since the model $\mathfrak{M}$ is expressed in $\mathbf{L}$ by the formula $A$, there is a homomorphism $h: \mathbb{Z} \times \mathbb{Z} \rightarrow W$ from $\mathfrak{M}$ to $M$. Denote $w_{m n}:=h(m, n)$, for $m, n \in \mathbb{Z}$.

Now put $t(m, n):=d$ iff $M, w_{m n} \models d$. This yields a total function $t: \mathbb{Z} \times \mathbb{Z} \rightarrow D$, since $M$ satisfies (Partitioning). Finally, let us verify the horizontal compatibility condition for $t$ (the vertical one is verified similarly).

Take any $m, n \in \mathbb{Z}$. Assume $m$ is even; the case of odd $m$ is considered similarly. Then we have $\langle m, n\rangle \prec\langle m+1, n\rangle$ (use the Right relation). By homomorphism, $w_{m n} R w_{m+1, n}$. Denote $i:=m \bmod 4$ and $j:=n \bmod 4$, then $\langle m, n\rangle \mid=p_{i j}$ and $\langle m+1, n\rangle \models p_{i \oplus 1, j}$. By homomorphism, $w_{m n} \models p_{i j}$ and $w_{m+1, n} \models p_{i \oplus 1, j}$. Denote $c:=t(m, n), d:=t(m+1, n)$, then $w_{m n} \models c$ and $w_{m+1, n} \models d$, by construction of $t$. We need to prove that $\langle c, d\rangle \in H$. To this end, we use that

$$
M, w_{m n} \models\left(p_{i j} \wedge c\right) \rightarrow \square\left(p_{i \oplus 1, j} \rightarrow \bigvee_{\left\langle c, d^{\prime}\right\rangle \in H} d^{\prime}\right),
$$

This implies $w_{m+1, n} \models d^{\prime}$, for some $d^{\prime} \in D$ with $\left\langle c, d^{\prime}\right\rangle \in H$. But the point $w_{m+1, n}$ satisfies (Partitioning) and so only one element of $D$ is true in it. Therefore, $d=d^{\prime}$ and $\langle c, d\rangle \in H$.

Lemmas 3.1 and 3.2 immediately imply that the logics GrIK4 and GrIS4 are globally undecidable. But since, for both logics, the reduction formula $A \wedge A_{\mathcal{D}}$ was the same, we can use the global variant of Lemma 1.2 to obtain the following result. 
Theorem 3.3. Every logic $\mathbf{L}$ with $\mathbf{G r I K} 4 \subseteq \mathbf{L} \subseteq$ GrIS4 is globally undecidable.

Proof. Indeed, the formula $\Phi_{\mathcal{D}}=A \wedge A_{\mathcal{D}}$, where the formula $A$ is built in Section 3.1 and $A_{\mathcal{D}}$ is built in the proof of Lemma 3.2, satisfies the conditions of Lemma 1.2, sinse

- the equivalence $(i) \Leftrightarrow$ (ii) for $\mathbf{L}_{1}=$ GrIK4 was proved in Lemma 3.2(a), which is applicable to this logic due to Lemma 3.1(a);

- the equivalence $(i) \Leftrightarrow(i i i)$ for $\mathbf{L}_{2}=$ GrIS4 was proved in Lemma 3.2(b), which is applicable to this logic due to Lemma 3.1(b).

\section{Local undecidability of GrIK4}

The proof follows the same pattern: we express (in the local sense) a grid-like model in the logic GrIK4, and then use this fact for reducing the domino problem. But the first part of the proof (expressing the grid) becomes more involved. The main difficulty is to enforce that all the points of the $\mathbb{Z} \times \mathbb{Z}$ grid depicted in Fig. 1 satisfy two conditions simultaneously: all they must be accessible from a single point and verify a certain modal formula. These conditions are interdependent: the former ensures the latter, and vice versa; so we need to break the cycle somewhere. This is what the Step Lemma proved below does.

\subsection{Expressing a grid}

We modify the model $\mathfrak{M}=(\mathbb{Z} \times \mathbb{Z}, \prec, \lambda)$ built in Section 3.1 (see Fig. 1 ) by adding a new point that "sees" all points of $\mathbb{Z} \times \mathbb{Z}$. Formally, consider the set of 17 propositional variables $\mathbb{P}=$ $\{E\} \cup\left\{p_{i j} \mid 0 \leqslant i, j \leqslant 3\right\}$. Let $\mathfrak{M}^{\prime}=\left(\{e\} \cup \mathbb{Z} \times \mathbb{Z}, \prec^{\prime}, \lambda^{\prime}\right)$ be a model, where $e \notin \mathbb{Z} \times \mathbb{Z}$,

$$
\prec^{\prime}=\prec \cup(\{e\} \times(\mathbb{Z} \times \mathbb{Z})),
$$

and the valuation $\lambda^{\prime}$ extends $\lambda$ to the new variable $E$ by $\lambda^{\prime}(E):=\{e\}$. Observe that $\mathfrak{M}^{\prime}$ is again a transitive model. Denote by $\mathfrak{M}_{\bullet}^{\prime}=\left(\{e\} \cup \mathbb{Z} \times \mathbb{Z}, \preccurlyeq^{\prime}, \lambda^{\prime}\right)$ the reflexive closure of the model $\mathfrak{M}^{\prime}$.

Denote $Z:=\bigvee_{i, j \in\{0,1,2,3\}} p_{i j}$. Now consider the formula

$$
A^{\prime}=E \wedge \diamond \top \wedge \square \neg E \wedge \square(A \wedge B),
$$

where $A$ is the conjunction of the formulas (1)-(4) built in Section 3.1 and $B$ is the conjunction of the following formulas:

$$
\begin{array}{ll}
\text { (5) } & \boxminus(Z \rightarrow \diamond E) \\
\text { (6) } & \diamond^{\leqslant 1} E
\end{array}
$$

Here comes the key lemma. It enables us to "build" points of the grid $\mathbb{Z} \times \mathbb{Z}$ one by one in any transitive model that satisfies the formula $A^{\prime}$ at some point (called root). When we build a new point, say $y$, of the grid, two cases are possible: if $y$ is $R$-accessible from the old one, say $x$, then $y$ is $R$-accessible from the root just by transitivity of $R$. However, if $y$ is $R^{-1}$-accessible from $x$, then the mere transitivity of $R$ does not help. In this case we use that $x$ satisfies the formulas (5) and (6), and this enables us to conclude that $y$ is $R$-accessible from the root.

Step Lemma. Let $M=(W, R, \theta)$ be a transitive model, $d R x, d \models A^{\prime}, 0 \leqslant i, j, k, \ell \leqslant 3$.

(forth) If $x \models p_{i j}$ and $\langle i, j\rangle \boxminus\langle k, \ell\rangle$, then there is $y \in W$ with $d R y, x R y$, and $y \models p_{k \ell}$.

(back) If $x \models p_{k \ell}$ and $\langle i, j\rangle \models\langle k, \ell\rangle$, then there is $y \in W$ with $d R y, y R x$, and $y \models p_{i j}$. 
Proof. Note that since $d \models A^{\prime}$ and $d R x$, we have $d \models E$ and $x \models A \wedge B$.

(forth) By (3) and $x \models p_{i j}$, we have $x \models \diamond p_{k \ell}$. Hence there is $y \in W$ such that $x R y$ and $y \models p_{k \ell}$. By transitivity of $R$, we have that $d R y$.

(back) By (3) and $x \models p_{k \ell}$, we have $x=\diamond p_{i j}$. Hence there is $y \in W$ such that $y R x$ and $y \models p_{i j}$. It remains to show that $d R y$. By (5), we have $x \models \boxminus(Z \rightarrow \diamond E)$, so that $y \models Z \rightarrow \diamond E$. Since $y \models Z$ (recall that $y \models p_{i j}$ ), we have $y \models \ominus E$. Therefore, there is $d^{\prime} \in W$ such that $d^{\prime} R y$ and $d^{\prime} \mid=E$. By transitivity, $d^{\prime} R y R x$ implies $d^{\prime} R x$. Thus, $x$ is accessible from two points, $d$ and $d^{\prime}$, that satisfy $E$. But $x=\ominus^{\leqslant 1} E$, by (6). Therefore, $d=d^{\prime}$ and hence $d R y$, as required.

Lemma 4.1. (a) The pointed model $\left(\mathfrak{M}^{\prime}, e\right)$ is expressible in GrIK4 (by the formula $A^{\prime}$ ).

(b) The pointed model $\left(\mathfrak{M}_{\bullet}^{\prime}, e\right)$ is expressible in GrIS4 (by the same formula $A^{\prime}$ ).

Proof. We prove only (a); the proof of (b) is similar. Since $\prec^{\prime}$ is transitive, $\mathfrak{M}^{\prime}$ is a GrIK4-model. It is not hard to see that $\mathfrak{M}^{\prime}, e \models A^{\prime}$. In particular, (4) holds in $\mathfrak{M}^{\prime}$, since the edges between the points of $\mathbb{Z} \times \mathbb{Z}$ are the same in $\mathfrak{M}^{\prime}$ and in $\mathfrak{M}$. It remains to prove that if $M=(W, R, \theta)$ is an arbitrary transitive model, $d \in W$, and $M, d=A^{\prime}$, then $\left(\mathfrak{M}^{\prime}, e\right) \hookrightarrow(M, d)$.

To this end, we will find a set of (not necessarily distinct) points $\left\{w_{m n} \in W \mid m, n \in \mathbb{Z}\right\}$ such that the mapping $h:(\{e\} \cup \mathbb{Z} \times \mathbb{Z}) \rightarrow W$ defined by $h(e)=d$ and $h(m, n)=w_{m n}$ is a homomorphism from $\left(\mathfrak{M}^{\prime}, e\right)$ to $(M, d)$.

Since $d \models A^{\prime}$, there is $w \in W$ such that $d R w$ and $w \models A \wedge B$. By (1), $w \models p_{i j}$ for some $i, j$. Without loss of generality, $w=p_{00}$. If, for example, $w=p_{12}$, then in 3 jumps we can reach a point that satisfies $p_{00}$ and is accessible from $d$, using the Step Lemma (see Fig. 1):

- since $\langle 1,2\rangle \Leftrightarrow\langle 1,1\rangle$, there is $x \in W$ with $w R x, x \models p_{11}$, and $d R x$;

- since $\langle 1,0\rangle \Leftrightarrow\langle 1,1\rangle$, there is $y \in W$ with $y R x, y \models p_{10}$, and $d R y$;

- since $\langle 0,0\rangle \Leftrightarrow\langle 1,0\rangle$, there is $z \in W$ with $z R y, z \models p_{00}$, and $d R z$.

So, we have a point $w_{00} \in W$ such that $d R w_{00}$ and $w_{00}=p_{00}$. Next, using the Step Lemma, we build a "horisontal axis" of points $\left\{w_{m, 0} \in W \mid m \in \mathbb{Z}\right\}$ that are accessible from $d$ (i.e., $\left.d R w_{m, 0}\right)$, linked by $R$-edges of interleaving directions (see Fig. 1):

$$
\ldots \stackrel{R}{\longleftarrow} w_{-2,0} \stackrel{R}{\longrightarrow} w_{-1,0} \stackrel{R}{\longleftarrow} w_{0,0} \stackrel{R}{\longrightarrow} w_{1,0} \stackrel{R}{\longleftarrow} w_{2,0} \stackrel{R}{\longrightarrow} \ldots
$$

and satisfy $w_{m, 0} \models p_{i, 0}$, where $i=m \bmod 4$. Likewise, we build a "vertical axis" of points $\left\{w_{0, n} \in W \mid n \in \mathbb{Z}\right\}$ that are linked in a similar way and satisfy $d R w_{0, n}$ and $w_{0, n} \models p_{0, j}$, where $j=n \bmod 4$. Finally, we complete the $\mathbb{Z} \times \mathbb{Z}$ grid as in Lemma3.1 (see Fig. 2): whenever we have two horizontal and two vertical edges, we complete them in a $3 \times 3$ grid using (4) and the Step Lemma.

Thus, the model $M$ contains a set of points $\left\{w_{m n} \in W \mid m, n \in \mathbb{Z}\right\}$ such that $d R w_{m n}$ and $w_{m n} \models p_{(m \bmod 4),(n \bmod 4)}$. Note that $w_{m n} \not \neq E$, for all $m, n$. The above construction implies that the mapping $h$ defined by $h(e)=d$ and $h(m, n)=w_{m n}$ is a homomorphism from $\left(\mathfrak{M}^{\prime}, e\right)$ to $(M, d)$, as required.

\subsection{Reducing the domino problem}

Lemma 4.2. Assume that the language of a logic $\mathbf{L}$ contains the modal operator $\diamond$.

(a) If the pointed model $\left(\mathfrak{M}^{\prime}, e\right)$ is expressible in $\mathbf{L}$, then $\mathbf{L}$ is undecidable.

(b) If the pointed model $\left(\mathfrak{M}_{\bullet}^{\prime}\right.$, e) is expressible in $\mathbf{L}$, then $\mathbf{L}$ is undecidable.

Proof. The proof repeats that of Lemma 3.2 with the following changes. Recall that, given a domino system $\mathcal{D}$, a formula $A_{\mathcal{D}}$ was built in that proof. Let $A^{\prime}$ be an $\mathbf{L}$-formula that expresses the pointed model $\left(\mathfrak{M}^{\prime}, e\right)$ (or $\left(\mathfrak{M}_{\bullet}^{\prime}, e\right)$, respectively) in $\mathbf{L}$. Denote $\Phi_{\mathcal{D}}^{\prime}:=A^{\prime} \wedge \square\left(Z \rightarrow A_{\mathcal{D}}\right)$. 
Claim. A domino system $\mathcal{D}$ tiles $\mathbb{Z} \times \mathbb{Z} \Longleftrightarrow$ the formula $\Phi_{\mathcal{D}}^{\prime}$ is satisfiable in $\mathbf{L}$.

This claim is proved as in Lemma 3.2, using the fact that, in the models $\mathfrak{M}^{\prime}$ and $\mathfrak{M}_{\bullet}^{\prime}$, it is exactly the points of $\mathbb{Z} \times \mathbb{Z}$ that are accessible from $e$ and satisfy $Z$.

So, the logics GrIK4 and GrIS4 are undecidable. Moreover, since the reduction formula $\Phi_{\mathcal{D}}^{\prime}$ was the same for both logics, we can use the local variant of Lemma 1.2 and obtain

Theorem 4.3. Every logic $\mathbf{L}$ with $\mathbf{G r I K} 4 \subseteq \mathbf{L} \subseteq$ GrIS4 is undecidable.

\section{$5 \quad$ Undecidability of the unrestricted DL $\mathcal{S I Q}$}

Description logics (DLs) are a well-known family of logic-based knowledge representation formalisms [1]. A rather expressive DL named $\mathcal{S H O \mathcal { Q }}$ serves as a logical underpinning for the Web Ontology Language OWL [10]. Here we focus on its fragment named $\mathcal{S I} \mathcal{Q}$. Both $\mathcal{S} \mathcal{I}$ and $\mathcal{S H O I Q}$ have a certain restriction on its syntax that guarantees decidability of reasoning. As we show below, if we omit this restriction, then reasoning in the resulting "unrestricted" $\mathcal{S I} \mathcal{Q}$ becomes undecidable, even if there is only one transitive role in the vocabulary. To the best of our knowledge, this is the first undecidability result for a fragment of $\mathcal{S H O \mathcal { O }}$ that does not involve the so-called role hierarchy (reflected by the letter $\mathcal{H}$ in the logic's name). Compare this with the undecidability result from [7] for the $\operatorname{logic} \mathcal{S H \mathcal { Q }}$, which has role hierarchy, but not inverse roles (and the proof needs 8 roles; a more subtle proof with only 3 roles was given in [9]).

In few words, the undecidable modal logic GrIK4 is (a notational variant of) a fragment

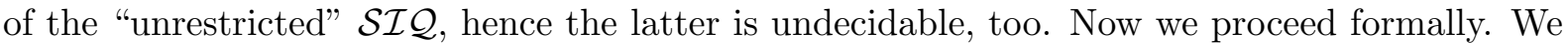
briefly recall the main definitions; for more information on DL, the reader is referred to $[1,10]$.

Concepts. The basic DL named $\mathcal{A L C}$ is a notational variant of the minimal multi-modal logic $\mathbf{K}_{m}$, as can be noticed by comparing the syntax for concepts of $\mathcal{A L C}$ and for formulas of $\mathbf{K}_{m}$ :

$$
\begin{aligned}
\text { concepts of } \mathcal{A L C}: \quad C, D::=\top|\perp| A_{k}|\neg C| C \sqcap D|C \sqcup D| \exists R_{i} C \mid \forall R_{i} . D \\
\text { formulas of } \mathbf{K}_{m}: \quad \varphi, \psi::=\top|\perp| p_{k}|\neg \varphi| \varphi \wedge \psi|\varphi \vee \psi| \diamond_{i} \varphi \mid \square_{i} \varphi
\end{aligned}
$$

Here $\left\{A_{1}, A_{2}, \ldots\right\}$ are concept names and $\left\{R_{1}, \ldots, R_{m}\right\}$ are role names; altogether, they form a vocabulary. Semantics of $\mathcal{A L C}$ concepts is identical to that for the corresponding formulas of $\mathbf{K}_{m}$. Many extensions of $\mathcal{A L C}$ are notational variants of extended modal logics; we only need the following two extensions of the $\mathcal{A L C}$ syntax:

$(\mathcal{I})$ inverse roles: concepts $\exists R_{i}^{-} . C$ and $\forall R_{i}^{-} . C$ are added to the syntax; they correspond to the inverse (or converse) modalities $\vartheta_{i} \varphi$ and $\boxminus_{i} \varphi$;

$(\mathcal{Q})$ qualified number restrictions: concepts $\geqslant k R_{i} . C$ (and $\geqslant k R_{i}^{-} . C$, if inverse roles are available), for all $k \geqslant 1$, are added to the syntax; they correspond to the (forward and backward) graded modalities $\diamond_{i}^{\geqslant k} \varphi$ and $\vartheta_{i}^{\geqslant k} \varphi$.

Terminologies. A terminology (or a TBox) is a finite set of axioms of the form $C \sqsubseteq D$, where $C$ and $D$ are arbitrary concepts. A model satisfies an axiom $C \sqsubseteq D$ if, for every point $x$ in it, if $C$ holds at $x$ then so does $D$; in modal logic, this amounts to saying that an implication $\varphi \rightarrow \psi$ is true in a model. A model is said to satisfy a TBox if it satisfies all its axioms. Besides the TBox axioms, we need an additional kind of axioms; they form a so-called RBox:

$(\mathcal{S})$ transitivity axioms $\operatorname{Tr}\left(R_{i}\right)$; they correspond to saying that the modality $\square_{i}$ is transitive, i.e., to considering the class of models in which the $i$-th relation is transitive. 
So, the constructors $(\mathcal{I})$ and $(\mathcal{Q})$ extend the syntax for concepts, while $(\mathcal{S})$ reduces the class of models. ${ }^{4}$ Let us call the DL $\mathcal{A L C}$ extended with these 3 features the unrestricted $\mathcal{S} \mathcal{I} \mathcal{Q}$. In the "restricted" $\mathcal{S} \mathcal{I} \mathcal{Q}$ (called just $\mathcal{S} \mathcal{I} \mathcal{Q}$ ), the following condition is imposed on the syntax:

only non-transitive roles $R_{i}$ are allowed in concepts of the form $\geqslant k R_{i} . C$ and $\geqslant k R_{i}^{-}$.C.

To be more precise, once we fix an RBox $\mathcal{R}$ and thus know which roles are asserted to be transitive and which are not, the qualified number restrictions are only allowed for non-transitive roles.

Reasoning. The following reasoning problems in DL are relevant to our paper:

- concept satisfiability (with respect to an RBox $\mathcal{R}$ ): given a concept $C$, decide if $C$ holds in at some point of some model (satisfying $\mathcal{R}$ ). This problem corresponds to the local satisfiability of modal formulas (in the class of frames satisfying the RBox $\mathcal{R}$ ).

- TBox consistency (with respect to an RBox $\mathcal{R}$ ): given a TBox $\mathcal{T}$, decide whether there is a model satisfying $\mathcal{T}$ (and $\mathcal{R}$ ). Any TBox can be equivalently rewritten into a single axiom of the form $T \sqsubseteq E$ : just take $E$ to be the conjunction of concepts $\neg C \sqcup D$ for all axioms $C \sqsubseteq D$ in $\mathcal{T}$. It is then clear that the TBox consistency problem corresponds to the global satisfiability of modal formulas (in the class of frames satisfying the RBox $\mathcal{R}$ ).

Intuitively, for a fixed RBox $\mathcal{R}$, reasoning in the "unrestricted" $\mathcal{S} \mathcal{I} \mathcal{Q}$ corresponds to reasoning with two groups of modalities that obey the modal logics GrIK and GrIK4, respectively; these modalities correspond to the non-transitive and transitive roles in $\mathcal{R}$. From this viewpoint, the "unrestricted" $\mathcal{S I} \mathcal{Q}$ corresponds to what is called the fusion (cf. [15]) of several copies of the logics GrIK and GrIK4. Therefore, for a non-empty RBox, GrIK4 is a (notational variant of a) fragment of $\mathcal{S I} \mathcal{Q}$. This observation implies the following result.

Theorem 5.1. In the "unrestricted" description logic $\mathcal{S} \mathcal{I}$, the problems of concept satisfiability (w.r.t. an RBox) and TBox consistency (w.r.t. an RBox) are undecidable. The result holds even with one transitive role in the vocabulary.

On the contrary, the ordinary (i.e., restricted) DL $\mathcal{S I \mathcal { Q }}$ corresponds to the fusion of several copies of the decidable modal logics GrIK and IK4. It is known that reasoning in $\mathcal{S I} \mathcal{Q}$, and even in a more expressive DL $\mathcal{S H O I \mathcal { Q }}$, is decidable [10], which agrees with the fact that decidability is preserved under taking the fusion of logics [15].

\section{Complexity of fragments of GrIK4}

There are three directions in which the modal logic $\mathbf{K}$ can be extended: one can add inverse modalities (I), or graded modalities $(\mathbf{G r})$, or the transitivity (4). The first two, $\mathbf{I}$ and $\mathbf{G r}$, extend the syntax of formulas, whereas the third one, $\mathbf{4}$, reduces the class of frames. Adding each feature has the effect of increasing the set of valid formulas. Since there are 8 possible combinations of these features, this gives us 8 logics between $\mathbf{K}$ and GrIK4.

Here we give a survey of the complexity results of the local and global satisfiability problems for these logics. Since DLs are closely related to these logics, as we explained above, we will use known results on complexity of reasoning in DLs in order to obtain complexity results for modal logics. The results are summarized in Table 1 and visualized in the two "cubes of logics".

\footnotetext{
${ }^{4}$ This corresponds, for a modal logic $\mathbf{L}=(\mathcal{L}, \mathcal{F})$, to extending the language $\mathcal{L}$ and reducing the class $\mathcal{F}$.
} 


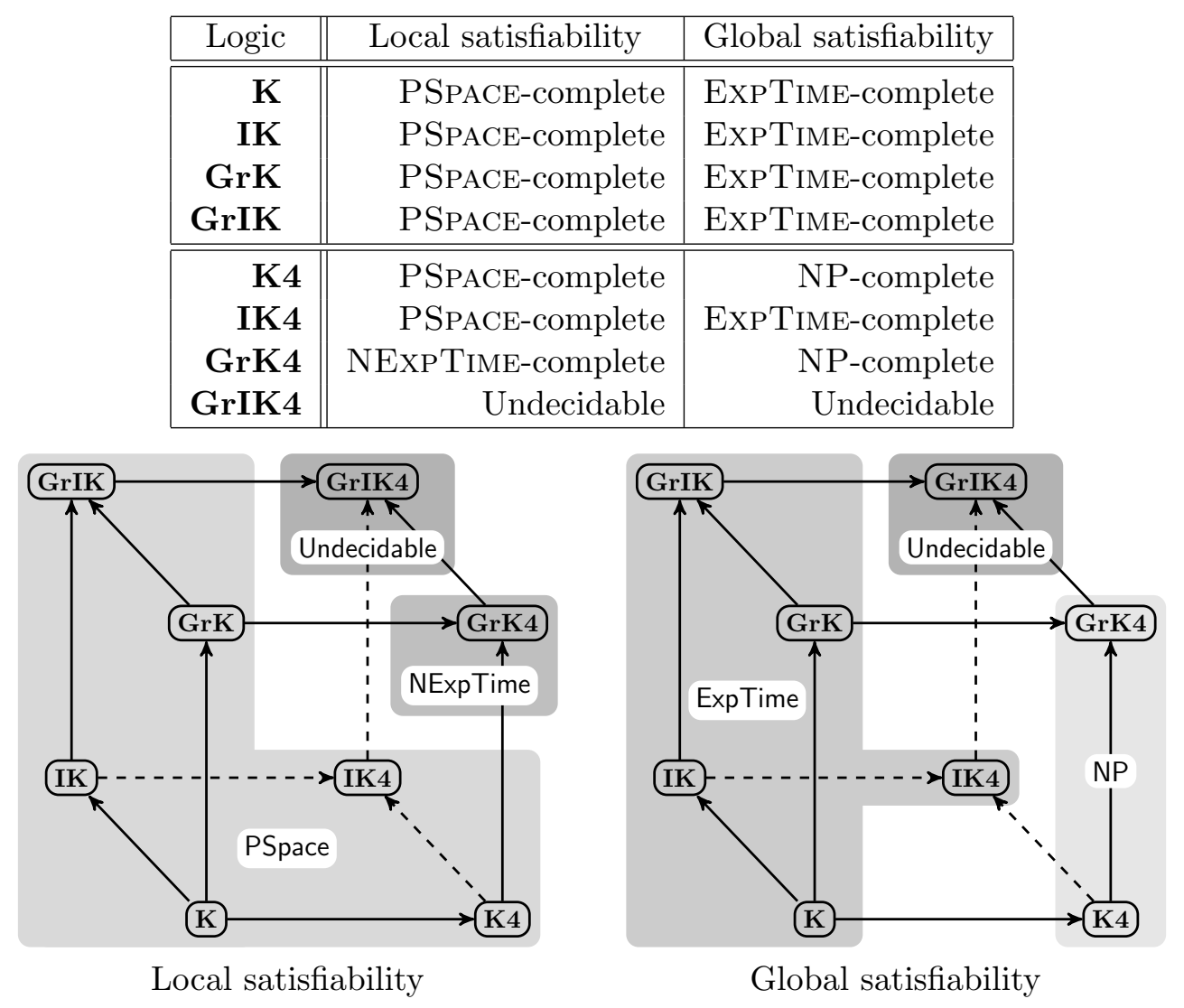

Table 1: Complexity of some logics between $\mathbf{K}$ and GrIK4.

Collections of many complexity results for modal and description logics can be found in the books [1, Ch. 3], [2, Ch.6], [3, Ch.4], [5, Ch.18], [6, Sect.1.6], and the theses [12, 14].

Let us say that a logic $\mathbf{L}$ is (locally or globally) $\mathcal{C}$-complete, for $\mathcal{C}$ a complexity class, if the problem of (local or global) satisfiability of formulas in $\mathbf{L}$ is $\mathcal{C}$-complete. Similarly, a DL will be called (locally or globally) $\mathcal{C}$-complete, if the problem of concept satisfiability or TBox consistency, respectively, for this DL is $\mathcal{C}$-complete. For brevity, we drop the words "a notational variant of" and simply say that a particular modal logic is a fragment of some DL. Below, we give explanations and references to all the results.

\section{Local complexity}

- The logics $\mathbf{K}$ and $\mathbf{K} 4$ are locally PSPACE-complete [11].

- The DL $\mathcal{A} \mathcal{L C} \mathcal{I} \mathcal{Q}$, which is $\mathcal{A L C}$ with $\mathcal{I}$ and $\mathcal{Q}$, is locally PSPACE-complete [14, Th. 4.29]. Hence so are its fragments $\mathbf{G r K}, \mathbf{I K}, \mathbf{G r I K}$, which contain $\mathbf{K}$ as a fragment; cf. [3, p. 186].

- The DL $\mathcal{S I}$, which is $\mathcal{S I} \mathcal{Q}$ without $\mathcal{Q}$, is locally PSPACE-complete [7, Th. 4.9]. Hence so is its fragment IK4 (also known as the temporal logic K4.t), of which K4 is a fragment.

- Recently, GrK4 (and GrS4) was shown to be locally NExpTime-complete [8].

- The modal logic GrIK4 is locally undecidable by our Theorem 4.3. 


\section{Global complexity}

- The logic $\mathbf{K}$ is globally ExPTime-complete [6, Th. 1.23].

- The (restricted) DL $\mathcal{S I} \mathcal{Q}$ (and even $\mathcal{S H I} \mathcal{Q}$ ) is globally ExpTIME-complete [14, Corol. 6.29]. Hence so are its fragments IK, GrK, GrIK, which also contain $\mathbf{K}$ as a fragment.

- Lemma 6.2 below shows that the logics K4 and GrK4 are globally NP-complete.

- The modal logic IK4 (which is K4.t) is globally ExPTime-complete [3, Ch. 4], [6, Sect. 1.6].

- The modal logic GrIK4 is globally undecidable by our Theorem 3.3.

\subsection{Global NP-completeness of K4 and GrK4}

Denote by GSat $(\mathbf{L})$ the set of $\mathbf{L}$-formulas that are globally $\mathbf{L}$-satisfiable. The antimonotonicity obviously holds: if two logics $\mathbf{L} \subseteq \mathbf{L}^{\prime}$ are in the same language, then $\operatorname{GSat}(\mathbf{L}) \supseteq \operatorname{GSat}\left(\mathbf{L}^{\prime}\right)$. Let us recall the facts that we use below.

- The logic S5 of frames with total relation is (locally and globally) NP-complete [11].

- The logic GrS5 is (locally and globally) NP-complete, too [8].

- The logic Ver of an irreflexive singleton frame is (locally and globally) NP-complete. Indeed, it is axiomatizable over $\mathbf{K}$ by the formula $\square \perp$, so in order to check if a given formula is (locally or globally) satisfiable in Ver, it suffices to replace all its subformulas of the form $\square \varphi$ with $T$ and check if the resulting propositional formula is satisfiable.

- The logic GrVer is (locally and globally) NP-complete, by the same argument, but with replacing all subformulas of the form $\diamond^{\geqslant k} \varphi$ with $\perp$.

In [13, Prop. 7] it was shown that GSat $(\mathbf{S} 4)=$ GSat(S5). Hence $\mathbf{S} 4$ is globally NPcomplete. Similarly, one can show that GSat $(\mathbf{G r S 4})=$ GSat $(\mathbf{G r S 5})$, so that GrS4 is globally NP-complete. By a slight modification of the argument from [13], we prove the following

Lemma 6.1. (a) GSat(K4) = GSat(S5) $\cup$ GSat(Ver),

(b) $\mathbf{G S a t}(\mathbf{G r K 4})=\mathrm{GSat}(\mathbf{G r S 5}) \cup \mathrm{GSat}(\mathbf{G r V e r})$.

Proof. (a) The inclusion $(\supseteq)$ is due to the antimonotonicity of GSat. To prove the inclusion $(\subseteq)$, take any formula $A$ that is true in some transitive model. It is known that the global satisfiability (and even a more general global consequence relation) in $\mathbf{K} \mathbf{4}$ is determined by finite frames (see, e.g., [6, Th. 1.21]). Hence $A$ is true in some finite transitive model $M$. The model $M$ then has a maximal element, i.e., a point $x_{0}$ such that $\forall y\left(x_{0} R y \Rightarrow y R x_{0}\right)$. Let $M^{\prime}$ be the submodel of $M$ generated by $x_{0}$. By the preservation property for modal formulas, $M^{\prime} \models A$. If $x_{0}$ has no successors, then $M^{\prime}$ is an irreflexive singleton model, hence $A \in \mathrm{GSat}(\mathrm{Ver})$. If $x_{0}$ has successors, then it is easily seen that the relation in $M^{\prime}$ is total, hence $A \in \mathrm{GSat}(\mathbf{S 5})$.

(b) Similarly, using the preservation property for graded modal formulas.

As an immediate consequence, we obtain the following result.

Lemma 6.2. For $\mathbf{K} 4$ and $\mathbf{G r K 4}$, the global satisfiability problem is NP-complete. 


\section{Conclusion}

In this paper, we considered the modal logic of transitive frames in the language that extends the ordinary modal language, which has the modality $\diamond$, with the inverse modality $\diamond$ and graded modalities, both forward $\diamond^{\geqslant n}$ and backward $\vartheta^{\geqslant n}$, for all $n \geqslant 2$. This logic is called GrIK4; its reflexive counterpart GrIS4 was also considered. For these logics, we proved that the problems of local and global satisfiability of formulas are undecidable. As a consequence, we proved that, in the "unrestricted" description logic $\mathcal{S I} \mathcal{Q}$, the problems of concept satisfiability and TBox consistency (w.r.t. an RBox) are undecidable, which gives a first example of an undecidable fragment of the unrestricted $\mathcal{S H O I} \mathcal{Q}$ that does not include role hierarchy. Additionally, for the logics between $\mathbf{K}$ and GrIK4, we gave a summary of complexity results for both problems.

For the aim of our undecidability proof, we introduced a ternary relation "a model is expressed in a logic by a modal formula", and its local analogue. It might be interesting to investigate model-theoretic properties of this notion, in various modal languages.

The logics for which we proved undecidability were formulated in the language $\{\diamond, \diamond\} \cup$ $\left\{\diamond^{\geqslant n}, \diamond^{\geqslant n} \mid n \geqslant 2\right\}$. A natural question arises whether the logic becomes decidable if we disallow the graded inverse modalities $\vartheta^{\geqslant n}, n \geqslant 2$. Notably, our undecidability proof did not use the whole infinite stock of the graded (forward and backward) modalities: actually, we only needed the modalities $\diamond^{\geqslant 2}$ and $\diamond^{\geqslant 2}$, or, more precisely, their negations $\diamond^{\leqslant 1}$ and $\diamond^{\leqslant 1}$. Then we can ask the same question for the language $\{\diamond, \diamond, \diamond \geqslant 2\}$. So, let us formulate the question explicitly.

Open problem. Decidability of the local and global satisfiability problems for the logics of (reflexive) transitive frames in the languages $\{\diamond, \diamond\} \cup\left\{\diamond^{\geqslant} \mid n \geqslant 2\right\}$ and $\{\diamond, \diamond, \diamond \geqslant 2\}$.

The logic with such a restricted syntax is in fact meaningful from the viewpoint of applications in knowledge representation. The transitive relation that we dealt with in this paper usually comes, in practice of ontological modeling, in the form of the (transitive) hasPart relation. It is then useful if the language allows one to count things (of a particular kind) that are parts of a given thing, by means of the graded modalities $\diamond^{\geqslant n}$ (or their DL counterparts, qualified number restrictions). On the contrary, it is usually less natural to count things of which a given thing is a part.

\section{References}

[1] Franz Baader, Diego Calvanese, Deborah McGuinness, Daniele Nardi, and Peter F. PatelSchneider, editors. The Description Logic Handbook: Theory, Implementation and Applications. Cambridge University Press, 2nd edition, 2007.

[2] Patrick Blackburn, Maarten de Rijke, and Yde Venema. Modal Logic, volume 53 of Cambridge Tracts in Theoretical Computer Science. Cambridge University Press, 2002.

[3] Patrick Blackburn, Johan van Benthem, and Frank Wolter, editors. Handbook of Modal Logic, volume 3 of Studies in Logic and Practical Reasoning. Elsevier, 2006.

[4] Egon Börger, Erich Grädel, and Yuri Gurevich. The Classical Decision Problem. Universitext. Springer, 2001.

[5] Alexander Chagrov and Michael Zakharyaschev. Modal Logic, volume 35 of Oxford Logic Guides. Oxford University Press, 1997. 
[6] Dov Gabbay, Agi Kurucz, Frank Wolter, and Michael Zakharyaschev. Many-Dimensional Modal Logics: Theory and Applications, volume 148 of Studies in Logic and the Foundations of Mathematics. Elsevier, 2003.

[7] Ian Horrocks, Ulrike Sattler, and Stephan Tobies. Practical reasoning for very expressive description logics. Logic Journal of the IGPL, 8(3):239-263, 2000.

[8] Yevgeny Kazakov and Ian Pratt-Hartmann. A note on the complexity of the satisfiability problem for graded modal logics. In Proc. of the 25th Annual IEEE Symposium on Logic in Computer Science (LICS 2010), volume IEEE Computer Society, pages 407-416, 2010.

[9] Yevgeny Kazakov, Ulrike Sattler, and Evgeny Zolin. How many legs do I have? Non-simple roles in number restrictions revisited. In N. Dershowitz and A. Voronkov, editors, Proc. of the 14th Int. Conf. on Logic for Programming, Artificial Intelligence, and Reasoning (LPAR 2007), volume 4790 of Lecture Notes in Computer Science, pages 303-317. Springer, 2007.

[10] Markus Krötzsch, Frantisek Simancik, and Ian Horrocks. A description logic primer. Computing Research Repository (CoRR), abs/1201.4089, 2012.

[11] Richard E. Ladner. The computational complexity of provability in systems of modal propositional logic. SIAM Journal on Computing, 6(3):467-480, 1977.

[12] Edith Spaan. Complexity of Modal Logics. PhD thesis, Department of Mathematics and Computer Science, University of Amsterdam, The Netherlands, 1993.

[13] Michael L. Tiomkin and Michael Kaminski. Nonmonotonic default modal logics. Journal of the ACM, 38(4):963-984, 1991.

[14] Stephan Tobies. Complexity results and practical algorithms for logics in knowledge representation. PhD thesis, LuFG Theoretical Computer Science, RWTH-Aachen, Germany, 2001.

[15] Frank Wolter. Fusions of modal logics revisited. In Marcus Kracht, Maarten de Rijke, Heinrich Wansing, and Michael Zakharyaschev, editors, Advances in Modal Logic, Volume 1, volume 87 of CSLI Lecture Notes, pages 361-379. CSLI Publications, Stanford, 1998. 